\title{
Strategies for introducing non-radioactive labels during the automated sequence analysis of nucleic acids
}

\author{
Gabor L. Igloi \\ Institut für Biologie III,Universität Freiburg Germany. Tel: 00497612032722 Fax: 00497612032787 \\ E-mail:igloi@oligo.biologie.uni-freiburg.de \\ The author's research was supported by the Deutsche Forschungsgemeinschaft (SFB388).
}

Rapid, large-scale genome as well as diagnostic DNA sequencing projects are, at present, dependent on the use of sensitive automated sequencers that rely on the detection of fluorescent signals. This emission is not an intrinsic property of the biomolecules but is a property of an optical label that must be incorporated before, during or after the sequence specific reaction. The choice of strategy, that is to be reviewed here, is a function of both the chemistry and the enzymology of the system as well as the nature of the fractionation and the physical parameters of the detection. One may differentiate beween the labelling of the primer, incorporation of the label during elongation or base specific termination with labelled terminators. In reviewing the development of each of these strategies, the conclusion is reached that, whereas labelled primers have universal applicability, the current generation of fluorescent terminators have in, conjunction with appropriate DNA polymerases, attained a refinement that strongly favours their use. However, since they are not available for all commercial sequencing systems, the alternative procedures are not merely of historic interest but are an essential component in DNA sequencing protocols. The possibility of automated direct sequence analysis of RNA has not been ignored completely.

Less than ten years after the milestone in molecular biology set by Maxam and Gilbert (1977) and Sanger et al (1977) with their development of DNA sequencing methods, means of automating at least some of the steps of these protocols were beginning to emerge (Ansorge et al., 1986; Smith et al., 1986, Prober et al., 1987). Fundamental to laying the foundation to large-scale sequencing, culminating in the Human Genome Project, was a replacement of conventional radioactive detection methods by optical systems much more amenable to automation (as well as reflecting the increasing environmental consciousness). Whereas hardware for stimulating the emission of fluorescence by biological molecules as well as highly sensitive systems for its detection was readily available, the lack of significant intrinsic fluorescent properties of any of the natural nucleobases was one key aspect which had to be considered at the outset. In contrast to radioactive labels, the introduction of an optical label - of necessity a chemical and physical perturbation of the system - leads to the questions of fundamental significance to both the biochemical and the physical part of the process. At what stage of the sequence reaction can one introduce a fluorescent tag ? Which fluorophore is compatible with the excitation/detection system ? The continued upgrading to new generations of the original instruments (Quesada and Zhang, 1996) and efforts to improve the properties of the dyes (Metzker et al., 1996;
Hung et al., 1996a,b; Lee et al., 1997) confirm that these considerations are still of relevance.

For the purposes of nucleic acid sequencing, one may consider two types of detection systems which are of importance. 1. Direct optical detection of a fluorescent signal. 2. Enzyme-coupled colourimetric or luminescent systems based on the biotin/avidin or on the digoxigenin/anti-digoxigenin interactions. Commercial fluorescence-based sequencers may be classified into two general types (Hunkapillar et al., 1991), which also dictate, to some extent, the choice of labelling strategies. On the one hand, a single fluorescent dye is used in all four base-specific reactions and the products are, in analogy to conventional radioactive sequencing, fractionated in four adjacent lanes of a polyacrylamide gel. Alternatively, four optically different dyes are incorporated such that the wavelength of the emission characterises the base. In this system the reaction products may be fractionated in a single lane or a single capillary.

\section{Sequence analysis of DNA}

\section{Reaction according to Sanger}

The chain termination procedure introduced by Sanger et al. (1977) is dependent on the use of a short oligonucleotide primer, complementary to a target sequence on the DNA, which is enzymatically elongated and subsequently basespecifically terminated. With the advent of chemical methods for the efficient synthesis of oligonucleotides (Caruthers et al., 1987) and the emergence automated DNA synthesisers in the early '80s, the Sanger method has become the method of choice for all DNA sequencing projects. For the detection of the products of this reaction, requiring the incorporation of a label, a number of options exist irrespective of whether radioactive isotopes, fluorescent dyes or other tags are used. The available choices are summarised schematically in Fig. 1.

\section{A. Labelling the primer (Fig. 1A)}

The $5^{\prime}$ terminus of the primer has been a popular target for the attachment of a variety of labels. It is, intuitively, a likely candidate since it is well separated from the $3^{\prime}$ end where enzymatic processes might be expected to be negatively affected by a chemical modification. On the other hand, as far as an enzymatic addition of a label is concerned, the 5' $\mathrm{OH}$ is particularly inert. One illustrative approach was an This paper is available on line at http://www.ejb.org 
attempted ligation of a phosphorylated deoxyoligonucleotide to an biotinylated trinucleotide terminating in ribo $\mathrm{C}$ (Kempe et al., 1985). In the presence of a total of $10 \mu \mathrm{g}$ RNA ligase and over a incubation period of $96 \mathrm{~h}$, a $20 \%$ yield of the desired biotinylated oligonucleotide was obtained. A better overall yield $(\sim 50 \%)$ has been obtained by Kinoshita et al. (1997) who used the adenylation reaction of RNA ligase to make a 5' - 5' intermediate in the presence of the aminogroup-bearing 3'aminoATP. This could then be modified further with succinimide derivatives. A 5'-5' linkage between a DNA oligomer and a ribonucleoside was also achieved chemically (Agrawal et al., 1986). The ribose unit could be oxidised and coupled to a semicarbazide derivative of the required label. The use of polynucleotide kinase, so well established in the field of radioactive labelling, can be eliminated as a direct way of adding a fluorescent label. An indirect approach of using this enzyme in the presence of the phosphorothioate analogue ATP $\mathrm{S}$ leads to a thiophosphorylated $5^{\prime} \mathrm{OH}$ which can then be chemically modified by alkylating reagents bearing the desired tag (Flick, 1995). This two stage procedure is the only practical direct alternative, to date, to the incorporation of the label 5'terminally solely by chemical methods. For certain applications where neither the corresponding phosphoramidite nor a reactive intermediate is available, as is the case for the infra red dyes (Middendorf et al., 1992), a ligation between a specific primer and a short oligonucleotide bearing the label and using a bridging complementary oligonucleotide template (Jang and Steffens, 1997) may be appropriate. Efforts at modifying pre-existing oligonucleotides pre-date the availability of automated synthesisers and were impractical for routine use since they required a number of steps followed by chromatographic purification (Kawashima and Chollet, 1985). Once the synthetic route via phosphoramidite chemistry had been established the way was open for the development of a series of intermediates which could be coupled to the oligonucleotides during synthesis and subsequently labelled with the desired tag. The initial development of automated DNA sequencers depended on the ability of adding a reactive $5^{\prime}$ terminal group to the oligonucleotide during its synthesis. This modification permitted a subsequent reaction of this residue with dye-thiocyanates, maleimides or, preferably, dye-succinimide esters. The phosphoramidite monomers used for adding the 5' amino or thio moiety (Smith et al., 1985; Connolly, 1985; Connolly, 1987) were indispensable until dye-phosphoramidites became available (Schubert et al., 1990; Cech et al., 1991; Theisen et al., 1992; Schubert et al., 1992) and are still essential for tagging with novel reagents. Unfortunately, while being ideal for labelling standard vector-specific primers, which are used repeatedly or even simultaneously as in multiple laser applications (Kambara et al., 1991; Wiemann et al., 1995), it is totally uneconomical to use commercial dye-phosphoramidite preparations for the synthesis of specific or "walking" primers unless one can co-ordinate a demand for large numbers of such oligonucleotides (Hawkins et al., 1992). A further argument against primers modified during chemical synthesis is that by blocking the 5' end one is automatically excluding other potential applications of this primer, such as 5 ' radiolabelling or phosphorylation prior to ligation.

Despite the use of lasers and CCD cameras, the sensitivity of detection of fluorescent DNA fragments is less than is achievable with isotopic labelling. This realisation underlies efforts at extending the sensitivity with the aim of extracting more data per sequencing run while reducing the amount of sample required. One can, on the one hand, simply incorporate multiple labels into a primer by using C-5 aminomodified uridines (Haralambidis et al., 1987; Brumbaugh et al., 1988) or an aminobutyl-1,3-propanediol modified backbone (Nelson et al., 1992) to achieve multiple dye substitutions. The addition of an internal fluorescent label, in addition to one at the $5^{\prime}$ terminus was found to enhance priming efficiency, although the hybridisation stability was reduced (Mineno et al., 1993). Branching phosphoramidites (Berlin et al., 1991) as well as multiple nucleotides bearing a fluorescein at the 1' position (Guzaev et al., 1994) should also be mentioned in this context. In the latter case, up to five modified bases could be introduced into a sequencing primer without effecting its function. However, the specificity of hybridisation cannot be expected to tolerate such extreme steric influences in all cases. Near infrared dyes are advantageous in this respect in that an increased sensitivity is attained due to a reduced background fluorescence (Middendorf et al., 1992) while at the same time allowing a simplification in the design of the hardware. In systems requiring the incorporation of four different dyes, the variation in the optical properties e.g. excitation wavelength or quantum yield of emission, as well as electrophoretic mobility variation makes certain demands on the hardware and on the evaluation software. A recent emphasis has, therefore, been on the development of energy transfer dyes. The physical basis behind this idea is the excitation of one fluorophore by the emitted light of a proximal second fluorophore with an overall enhancement of the emission intensity (Ju et al., 1995b). These dyes, initially based on the energy transfer from excited fluorescein to fluorescein and rhodamine derivatives, were positioned internally within the primer sequence with the donor placed at the 5 ' end ( $\mathrm{Ju}$ et al., 1995a,b). Cyanine-based energy transfer dyes, being optically superior in stability and quantum yield, were attached analogously to the primer (Hung et al., 1996a). The development of a family of uniformly migrating dye-primers using BODIPY dyes as donors (Metzker et al., 1996) with the internal fluorescein/rhodamine acceptors also addressed the problem of electrophoretic migration variability. A more flexible version of these constructs, using a polymeric spacer between donor and acceptor (Ju et al., 1996) and thus not being constrained by sequence requirements, would seem to be generally applicable, while still being dependent on de novo chemical synthesis of the labelled primer. Dye pairs (modified dichlororhodamine and fluorescein) coupled directly to each other and subsequently to amino-modified primers (Lee et al., 1997) have been described.

Following early proposals (Murasugi and Wallace, 1984) of the addition of a biotin label to the 3 ' end of a primer by a template-directed primer extension, there has been a general neglect of this potential labelling location. Template independent addition of nucleotides is the natural reaction of terminal deoxynucleotidyl transferase (TdT). However, for the purposes of introducing a single specific label at the 3' terminus the natural polymerisation activity of this enzyme must be restricted. Kössel and Roychoudhury (1971) initially described the limited elongation of DNA when riboNTP's replaced dNTP's in this reaction. Kumar et al. (1988) reported the addition of a limited number of modified rATP to oligonucleotides, while Alexandrova et al. (1991) noted that deoxynucleotides labelled at the 5 position with fluorescein were inhibitory to TdT resulting in the incorporation of one or two nucleotides. A combination of 
ribo- and fluorescein- (or biotin)-modified triphosphates was finally shown to give the desired substrate that could be efficiently used by TdT but that restricted the elongation of a sequencing primer to a single nucleotide (Igloi and Schiefermayr, 1993). Initially limited to the commercially available uridine derivative, the procedure was subsequently extended to fluorescein-ATP and -CTP (Igloi, 1996a)(essential for retaining the complementarity of the $3^{\prime}$ terminal nucleotide) and remains to date the only direct enzymatic, template independent method for labelling preexisting primers. Despite the proven advantages of this enzymatic procedure in terms of speed and economy during a genome project (Maier et al., 1995), Markiewicz et al. (1997), apparently unaware of previous publications, described the chemical synthesis of $3^{\prime}$ end labelled primers and their application in DNA sequencing.

Automated sequencing hardware based on the detection of fluorescence suffers the disadvantage of a high initial outlay of funds. An alternative, avoiding radioactive methods, which has been shown to be capable of producing high volume data (Pohl and Maier, 1995) is sequencing by direct blotting. During this procedure, whether with automated colour development (Patel and Nash, 1995) or not, (Pohl and Beck, 1987) the products of a gel-electrophoretic fractionation are blotted onto a membrane and visualised by an enzyme linked assay. Frequently, one component of the detection system, such as biotin (Patel and Nash, 1995; Olesen et al., 1993; Creasy et al, 1991; Martin et al., 1991; Beck, 1987) or digoxigenin (Richterich methods 1993) is borne by the sequencing primer while the detection is induced by binding streptavidin- or anti-digoxigenin-coupled phosphatase in the presence of a colourimetric or chemiluminescent substrate. Alternatively, and avoiding the disadvantages of having to label a primer, Purschke and Schäfer (1996) proposed the use of unlabelled primers followed by hybridisation of the blotted sequence pattern with a digoxigenin labelled probe. The probe may be a PCR product, incorporating digoxigenin, or a DNA strand labelled by one of a variety of methods available for providing hybridisation probes (Kessler, 1995).

\section{B. Labelling the growing DNA chain (Fig. 1B).}

Conventional radioactive sequencing protocols almost without exception utilise the incorporation of a labelled $\mathrm{dNTP}$ as the basis of the detection. The direct transfer of this concept to non-isotopic systems is not trivial. One would anticipate that a bulky modification to the triphosphate might influence the system at several stages. a) The dNTP may no longer be an effective substrate for the DNA polymerase b) If incorporated, sequences requiring multiple adjacent labelled nucleotides may lead to chain termination c) The electrophoretic migration of the fragments would be perturbed in an unpredictable fashion, depending on the number of labels incorporated. Surprisingly and despite these misgivings, the use of fluorescein-dUTP, believed to bring about a multiple labelling, was reported to give unambiguous sequences (Voss et al., 1991) using T7 DNA polymerase although the theoretical basis remained unclear. The use of fluorescein dATP further boosted the read length obtained (Wiemann et al., 1995). The variation in the quality of the sequence noted during routine use (Hawkins et al., 1992) was clarified somewhat after the detailed demands made on the primer and the target sequences were revealed (Wiemann et al., 1995) and refined (Wiemann et al., 1996). A screening of the polymerases, which had in the meantime become a target for genetic manipulation (Tabor and Richardson, 1995) showed that by no means all enzymes routinely used in sequencing would accept fluorescent dNTPs as substrates (Voss et al., 1997). In particular, only two of the thermostable DNA polymerases, used in the much favoured cycle sequencing protocol (Carothers et al., 1989), could be induced to incorporate these analogues. However, some other dye-labelled dNTPs, of relevance for the latest generation of automated sequencers using red laser excitation could be added to the repertoire. Previously, tetramethylrhodaminedATP had been introduced in the labelling step by Stark et al. (1994) in proposing a capillary electrophoretic two-label peak-height encoded DNA sequencing system. The internal label protocol was extended to an infrared dye labelled dATP (Steffens et al., 1995) and to digoxigenin-dATP (Pohl and Maier, 1995). Sequences comparable to those obtained with terminally labelled primers were obtained underlining the previous hypothesis of Wiemann et al.(1995) that only a single labelled nucleotide is incorporated into the growing DNA chain during the labelling stage of the reaction. With this explanation for the homogeneous migration of the labelled fragments comes the realisation that one is, in fact, observing a 3'-terminal primer labelling and not the continuous internal incorporation of the label. It is then, apart from the experimental details, related to the limited primer labelling strategy of DeBellis et al. (1995) using fluoresceindUTP and T7 DNA polymerase and hardly different from the terminal transferase mediated labelling of the primer (Igloi and Schiefermayr, 1993) described above. A labelling reaction using a DNA polymerase with a fluorescent dNTP plus low concentrations of unlabelled triphosphates is usually followed by the termination reaction, except in the special case of hexamer primers where a low temperature elongation must precede the labelling reaction (Hou and Smith 1993). Thus, instead of the template independent elongation of the primer with terminal transferase, requiring merely a knowledge of the immediately following base to ensure complementarity of the added base, complex rules of primer design must be followed to permit such "internal labelling" as to give the desired high quality sequence (Wiemann et al., 1995). As far as fluorescein-labelled nucleotides are concerned, there is neither an economic, nor a time or labour saving advantage for the internal approach. On the other hand and in contrast to the substrate selectivity of terminal transferase (Igloi 1996a), the remarkable tolerance of DNA polymerases towards modified nucleotides (Voss et al., 1997) provides a useful pathway to template dependent incorporation of non-fluorescein dNTPs.

\section{Labelling the termination (Fig. 1C).}

The conceptually most satisfying approach to the introduction of a label during the Sanger sequencing protocol is via a labelled terminating dideoxyNTP. Using such fluorescent analogues bypasses the difficulties of labelling the primer or of designing the primer for a successful enzymatic internal labelling. The interpretation of the sequence pattern is simplified by the visualisation of only those products arising out of a defined enzymatic termination, rather than from a random fragmentation, reduces the background and facilitates the automated evaluation. As in the case of four differently labelled primers (Smith, et al., 1986), the use of labelled terminators permits a "four-dye/one lane" approach whereby the products of the sequencing reaction can be fractionated in a single lane of a 
slab gel or in a single or multiple capillaries (Quesada and Zhang, 1996), using the spectral properties of the dyes to differentiate between bases. This not only eliminates interlane variation while increasing the throughput but by using labelled terminators provides the only possibility of performing the complete sequencing reaction in a single reaction vessel. These advantages were appreciated at the outset of the development of automated sequencers (Prober et al., 1987) at which time a set of fluorescein-based derivatised dideoxynucleotides was introduced. Despite the elegance of this approach, in practice several non-trivial problems involving the enzymology of the system arose. Dideoxynucleotides are, as such, poor substrates for most DNA polymerases. The fluorescein modified terminators were accepted by the enzyme (AMV reverse transcriptase) only at very high ddNTP concentrations resulting, despite extensive purification steps, in high background as well as expense. In addition, the set of dye-terminators could not be directly transferred to other polymerase systems but required an optimisation. Thus, for Taq DNA polymerase, essential for performing the cycle sequencing protocol, a set of rhodamine-based terminators was developed (Lee et al., 1992) which produced acceptable sequences, when used in conjunction with 2'-deoxyribonucleoside 5'-O-(1thiotriphosphates). The intrinsic peak height variation due to sequence dependent differential incorporation of the terminators by the Taq DNA polymerase was recognised and in an effort to utilise the processivity of T7 DNA polymerase to attain more even signal patterns a new set of labelled terminators was designed (Lee et al., 1992). Nevertheless, in order to maintain the convenience of the cycle sequencing system, a detailed analysis of the peak variation with respect to the sequence context provided a collection of empirical rules for the interpretation of Taq DNA polymerasegenerated patterns (Parker 1995). The difficulties of computer-based recognition of such variable signal strengths was underlined at this time by the conclusions of a comparative survey which found that dye-primer protocols could provide longer reliable information than dyeterminator systems (Naeve et al., 1995). This was a rather disappointing culmination of a decade of chemical effort in manipulating the ddNTPs in order to overcome the intrinsic biological barriers of the DNA polymerases. The breakthrough which was essential for increasing the accuracy of automated base-calling and any further development in the technology of automated sequencing was provided by approaching the problem from the enzymological aspect. By establishing the molecular basis for the recognition of ddNTPs by DNA polymerase (Tabor and Richardson, 1995) it now became possible to manipulate the enzyme genetically to such an extent that not only ddNTPs but also dye-labelled ddNTPs became rather good substrates. Further tuning of the dye component of the terminators now provided dichlororhodamine and, in particular, coupled fluoresceinrhodamine energy transfer dye-terminators (Rosenblum et al., 1997). With this development the use of dye terminators has finally come of age and there is now no reason why it should not become the method of choice in future electrophoresis-based fluorescent automated sequencing.

\section{Chemical cleavage reaction}

The importance of DNA sequencing by the specific chemical degradation method of Maxam and Gilbert (1977) has over the years, and since the availability of synthetic oligonucleotides, steadily decreased. While not being banished completely from the sequencers repertoire, it is now of significance for only a few special applications. These include the characterisation of very short DNA (e.g. oligonucleotides) and highly GC rich structures which may not be resolved by enzymatic methods. Fluorescein, when attached to DNA, has been shown using model oligonucleotides to survive the modification and cleavage reactions commonly used (Ansorge et al., 1988). Protocols for longer DNA have also been reported (Rosenthal et al., 1990) for which the fluorescent label has been introduced by initially elongating a fluorescent primer in a template dependent manner and subsequently sequencing the fluorescent strand. This approach would appear to be somewhat circuitous and does not demonstrate the advantages of the chemical cleavage reaction over the Sanger protocol. Not only is a fluorescent primer required, but its enzymatic elongation in those cases where the chemical degradation method would be favoured, i.e. GC rich templates, would be as problematic as if the Sanger method had been applied directly. A methodological simplification, making use of magnetic bead capture for the purification steps (Ohara et al., 1997) relies, however, on the availability of ' 5' biotin/5' fluorescein doubly labelled primers for labelling of the target DNA by PCR and subsequent solid phase capture. Richterich (1989) added a biotin label to DNA by endfilling a restriction site. Chemical degradation of this DNA, followed by blotting and colourimetric detection supplied satisfactory sequence information.

\section{Sequence analysis of RNA}

The direct sequence determination of RNA is limited to rather specialised applications where the pathway via cDNA sequencing is not available, e.g. small RNAs. However, the use of non-radioactive labels and automation using "DNA" sequencers has been demonstrated effectively. Analogously to the Sanger procedure, Bauer (1990) used fluorescein ddNTPs in a chain terminating reverse transcription reaction, while fluorescein labelled primers targeted at known sequence regions or at a previously ligated 3 ' tail have in conjunction with AMV reverse transcriptase also given clear sequences (GLI, unpublished results).

Sequencing of RNA by specific cleavage whether enzymatically or chemically requires the introduction of a terminal label. As in the case of DNA primers, either terminus may be modified but in the case of RNA, the choice of enzymatic reactions is limited to the use of RNA ligase. Biotin attached to $\mathrm{pCp}$ has been ligated to tRNA and reported to give satisfactory chemiluminescent sequence patterns after specific enzymatic degradation (Matsuki et al., 1993). Analogously, natural RNA's can be labelled with pSEEp, a fluorescent ribo-dinucleotide (Igloi 1996b). At the 5 ' end, an intermediate of the ligation reaction may be isolated and by using 3' aminoATP as the energy source, the adenylate may be chemically modified at the $3^{\prime}$ amino group to attach the desired label (Kinashita et al., 1997). Alternatively, periodate oxidation of the 3' terminal ribose permits a condensation reaction with an appropriately modified fluorescent label (Wu et al., 1996) and yields characteristic sequence patterns when digested with ribonucleases. In treating RNA with chemical reagents one should, nevertheless, be aware of possible side reactions. In the case of both of the latter labelling schemes, modified bases, which occur frequently in RNA, could well be 
affected and lead to the fluorescent tagging of internal positions.

\section{References}

Agrawal, S., Christodoulou, C. and Gait, M.J. (1986). Efficient methods for attaching non-radioactive labels to the 5 ' ends of synthetic oligodeoxyribonucleotides. Nucleic Acids Research 14:6227-6245

Alexandrova, L.A., Lukin, M.A., Victorova, L.S. and Rozovskaya, T. (1991). Enzymatic incorporation of fluorescent labels into oligonucleotides. Nucleic Acids Symposium Series 24:277.

Ansorge, W., Rosenthal, A., Sproat, B., Schwager, C., Stegemann, J. and Voss, H. (1988). Non-radioactive automated sequencing of oligonucleotides by chemical degradation. Nucleic Acids Research 16:2203-2206

Ansorge, W., Sproat, B.S., Stegemann, J. and Schwager, C. (1986). A non-radioactive automated method for DNA sequence determination. Journal of Biochemical and Biophysical Methods 13:315-323.

Bauer, G.J. (1990). RNA sequencing using fluorescentlabeled dideoxynucleotides and automated fluorescence detection. Nucleic Acids Research 18:879-884.

Beck, S. (1987). Colorimetric-detected DNA sequencing. Analytical Biochemistry 164:514-520.

Berlin, Y.A., Korshun, V.A. and Boreskov, Y.G. (1991). Multiple non-radioactive labeling of oligonucleotides. Nucleic Acids Symposium Series 25:85-86.

Brumbaugh, J.A., Middendorf, L.R., Grone, D.L. and Ruth, J.L. (1988). Continuous, on-line DNA sequencing using oligodeoxynucleotide primers with multiple fluorophores. Proceedings of the National Academy of Sciences USA 85:5610-5614.

Carothers, A.M., Urlaub, G., Mucha, J., Grunberger, D. and Chasin, L.A. (1989). Point mutation analysis in mammalian gene: rapid preparation of total RNA, PCR amplification of cDNA and Taq sequencing by a novel method. BioTechniques 7:494-499

Caruthers, M.H., Barone, A.D., Beaucage, S.L., Dodds, D.R., Isher, E.F.F., McBride, L.J., Matteucci, M., Stabinsky, Z. and Tang, J.-Y. (1987). Chemical synthesis of deoxyoligonucleotides by the phosphoramidite method. Methods in Enzymology 154:287-313.

Cech, D., Schubert, F. and Möller, U. (1991). Nonradioactive labelling of oligonucleotides with prefunctionalized phosphoramidites or $\mathrm{H}$-phosphonates during automated synthesis. Nucleic Acids Symposium Series 24:205-206.

Connolly, B.A. (1985). Chemical synthesis of oligonucleotides containing a free sulphydryl group and subsequent attachment to thiol specific probes. Nucleic Acids Research 13:4485-4502.
Connolly, B.A. (1987). The synthesis of oligonucleotides containing a primary amino group at the 5' terminus. Nucleic Acids Research 15:3131-3139.

Craesey, A., D'Angio, L. Jr., Dunne, T.S., Kissinger, C., O'Keefe, T., Perry-O'Keefe, H., Moran, L.S., Roskey, M., Schildkraut, I., Sears, L.E. and Slatko, B. (1991). Application of a novel chemiluminescence-based DNA detection method to single-vector and multiplex DNA sequencing. BioTechniques 11:102-109.

DeBellis, G., Pergolizzi, R., Debernardi, S., Invernizzi, L. and Luzzana, M. (1995). Fluorescence-based automated DNA sequencing by limited primer labeling. BioTechniques 19:66-70.

Flick, P.K.(1995). DNA sequencing by non-isotopic methods. In:Non-isotopic probing, blotting and sequencing. 2 nd.ed.L.J.Kricka. SanDiego, Academic Press, pp.475-492.

Guzaev, A., Azhayeva, E., Hovinen, J., Azhayev, A. and Lönnberg, H. (1994). Synthesis and primer properties of oligonucleotides containing 3'-deoxypsicothymidine units, labeled with fluorescein at their 1'-position. Bioconjugate Chemistry 5:501-503.

Haralambidis, J., Chai, M. and Tregear, G.W. (1987). Preparation of base-modified nucleosides suitable for nonradioactive label attachment and their incorporation into oligodeoxyribonucleotides. Nucleic Acids Research 15:48574876.

Hawkins, T.L., Du, Z., Halloran, N.D. and Wilson R.K. (1992). Fluorescent chemistries for automated primerdirected DNA sequencing. Electrophoresis 13:552-559.

Hou, W. and Smith, L.M. (1993) Fluorescence-based DNA sequencing with hexamer primers. Nucleic Acids Research 21:3331-3332.

Hung, S.-C., Ju, J., Mathies, R.A. and Glazer, A.N. (1996a). Cyanine dyes with high absorption cross section as donor chromophores in energy transfer primers. Analytical Biochemistry 243:15-27.

Hung, S.-C., Ju, J., Mathies, R.A. and Glazer, A.N. (1996b). Energy transfer primers with 5- or 6-carboxyrhodamine-6G as acceptor chromophores. Analytical Biochemistry 238:165170.

Hunkapillar, T., Kaiser, R.J., Koop, B.F. and Hood, L. (1991). Large-scale and automated DNA sequence determination. Science 254:59-67.

Igloi, G.L. (1996a). Substrate properties of fluorescent ribonucleotides in the terminal-transferase-catalyzed labelling of DNA sequencing primers. BioTechniques 21: 1084-1092.

Igloi, G.L. (1996b). Non-radioactive labeling of RNA. Analytical Biochemistry 233:124-129.

Igloi, G.L. and Schiefermayr, E. (1993). Enzymatic addition of fluorescein- or biotin-riboUTP to oligonucleotides results in primers suitable for DNA sequencing and PCR. BioTechniques 15:486-497. 
Jang, G.Y. and Steffens, D.L. (1997). Ligation mediated fluorescent labeling of DNA sequencing primers. Nucleic Acids Research 25:922-923.

Ju, J., Glazer, A.N. and Mathies, R.A. (1996). Cassette labeling for facile construction of energy transfer fluorescent primers. Nucleic Acids Research 24:1144-1148.

Ju, J., Kheterpal, I., Scherer, J.R., Ruan, C., Fuller, C.W., Glazer, A.N. and Mathies, R.A. (1995a). Design and synthesis of fluorescence energy transfer dye-labeled primers and their application for DNA sequencing and analysis. Analytical Biochemistry 231:131-140.

Ju, J., Ruan, C., Fuller, C.W., Glazer, A.N. and Mathies, R.A. (1995b). Fluorescent energy transfer dye-labeled primers for DNA sequence analysis. Proceedings of the National Academy of Sciences USA 92:4347-4351.

Kambara, H., Nagai, K. and Hayasaka, S. (1991). Real time automated simultaneous double-stranded DNA sequencing using two-color fluorophore labeling. Bio/technology 9:648651.

Kawashima, E.H. and Chollet, A. (1985). Biotin labeled synthetic oligodeoxyribonucleotides: chemical synthesis and uses as hybridization probes. Nucleic Acids Research 13:1529-1541.

Kempe, T., Sundquist, W.I., Chow, F. and Hu, S.-L. (1985). Chemical and enzymatic biotin-labeling of oligodeoxyribonucleotides. Nucleic Acids Research 13:4557.

Kessler, C. (1995). Methods for nonradioactive labeling of nucleic acids. In:Non-isotopic probing, blotting and sequencing. 2 nd.ed.L.J.Kricka. SanDiego, Academic Press, pp.41-109.

Kinoshita, Y., Nishigaki, K. and Husimi, Y. (1997). Fluorescence-, isotope- or biotin-labeling of the 5'-end of single-stranded DNA/RNA using T4 RNA ligase. Nucleic Acids Research 25:3747-3748.

Kössel, H. and Roychoudhury, R. (1971). Synthetic polynucleotides. The terminal addition of riboadenylic acid to deoxyoligonucleotides by terminal deoxynucleotidyl transferase as a tool for the specific labelling of deoxyoligonucleotides at the 3 ' ends. European Journal of Biochemistry 22: 271-276.

Kumar, A., Tchen, P., Roullet, F. and Cohen, J. (1988). Nonradioactive labeling of synthetic oligonucleotide probes with terminal deoxynucleotidyl transferase. Analytical Biochemistry 169:376-382.

Lee, L.G., Connell, C.R., Woo, S.L., Cheng, R.D., McArdle, B.F., Fuller, C.W., Halloran, N.D. and Wilson, R.K. (1992). DNA sequencing with dye-labeled terminators and T7 DNA polymerase: effect of dyes and dNTPs on incorporation of dye-terminators and probability analysis of termination fragments. Nucleic Acids Research 20:2471-2483.

Lee, L.G., Spurgeon, S.L., Heiner, C.R., Benson, S.C., Rosenblum, B.B., Menchen, S.M., Graham, R.J., Constantinescu, A., Upadhya, K.G. and Cassel, J.M. (1997).
New energy transfer dyes for DNA sequencing. Nucleic Acids Research 25, 2816-2822.

Maier, R.M., Neckermann, K., Igloi, G.L. and Kössel, H. (1995).Complete sequence of the maize chloroplast genome: gene content, hotspots of divergence and fine tuning of genetic information by transcript editing. Journal of Molecular Biology 251: 614-628.

Markiewicz, W.T., Gröger, G., Rösch, R., Zebrowska, A., Markiewicz, M., Klotz, M., Hinz, M., Godzina, P. and Seliger, H. (1997). A new method of synthesis of fluorescently labelled oligonucleotides and their application in DNA sequencing. Nucleic Acids Research 25:3672-3680.

Martin, C., Bresnick, L., Juo, R.-R.., Voyta, J.C. and Bronstein, I. (1991). Improved chemiluminescent DNA sequencing. BioTechniques 11:110-113.

Matsuki, T., Ito, R., Yokogawa, T. and Nishikawa, K. (1993). Sequence analysis of low-molecular-weight RNAs by the use of non-radioactive labeling. Nucleic Acids Symposium Series 29: 75-76.

Maxam, A.M. and Gilbert, W. (1977). A new method for sequencing DNA. Proceedings of the National Academy of Sciences USA 74:560-564.

Metzker, M.L., Lu, J. and Gibbs, R.A. (1996). Electrophoretically uniform fluorescent dyes for automated DNA sequencing. Science 271:1420-1422.

Middendorf, L.R., Bruce, J.C., Bruce, R.C., Eckles, R.D., Grone, D.L., Roemer, S.C., Sloniker, G.D., Steffens, D.L., Sutter, S.C., Brumbaugh, J.A. and Patonay, G. (1992). Continuous, on-line DNA sequencing using a versatile infrared scanner/electrophoresis apparatus. Electrophoresis 13:487-494.

Mineno, J., Ishino, Y., Ohminami, T. and Kato, I. (1993). Fluorescent labeling of a DNA sequencing primer. DNA Sequence 4:135-141.

Murasugi, A. and Wallace, R.B. (1984). Biotin-labeled oligonucleotides: enzymatic synthesis and use as hybridisation probes. DNA 3:269-277.

Naeve, C.W., Buck, G.A., Niece, R.L., Pon, R.T., Robertson, M. and Smith, A.J. (1995). Accuracy of automated DNA sequencing: a multilaboratory comparison of sequencing results. BioTechniques 19:448-453.

Nelson, P.S., Kent, M. and Muthini, S. (1992). Oligonucleotide labeling methods 3. Direct labeling of oligonucleotides employing a novel, non-nucleosidic, 2aminobutyl-1,3-propanediol backbone. Nucleic Acids Research 20:6253-6259.

Ohara, R., Tanaka, A. and Ohara, O. (1997). Automated fluorescent DNA sequencing by a simplified solid-phase chemical sequencing method. BioTechniques 22:653-656.

Olesen, C.E.M., Martin, C.S. and Bronstein, I. (1993). Chemiluminescent DNA sequencing with multiplex labeling. BioTechniques 15:480-485. 
Parker, L.T., Deng, Q., Zakeri, H., Carlson, C., Nickerson, D.A. and Kwok, P.Y. (1995). Peak height variation s in automated sequencing of PCR products using dye-terminator chemistry. BioTechniques 19:116-121.

Patel, A. and Nash, B. (1995). Innovations in non-isotopic DNA sequencing: using an electrotransfer unit to blot sequencing gels and an automated membrane processor for detecting DNA sequences. BioTechniques 18:328-333.

Pohl, F. M. and Beck, S. (1987). Direct transfer electrophoresis used for DNA sequencing. Methods in Enzymology 155:250-259.

Pohl, T.M. and Maier, E.(1995). Sequencing 500kb of yeast DNA using a GATC 1500 direct blotting electrophoresis system. BioTechniques 19:482-486.

Prober, J.M., Trainor, G.L., Dam, R.J., Hobbs, F.W., Robertson, C.W., Zagursky, R.J., Cocuzza, A.J., Jensen, M.A. and Baumeister, K. (1987). A system for rapid DNA sequencing with fluorescent chain-terminating dideoxynucleotides. Science 238:336-341.

Purschke, W.G. and Schäfer, G. (1996). An alternative to digoxigenin-labeled primers for manual non-radioactive sequencing allows reading of more than 700 bases. Analytical Biochemistry 238:98-100.

Quesada, M.A. and Zhang, S. (1996). Multiple capillary DNA sequencer that uses fiber-optic illumination and detection. Electrophoresis 17:1841-1851.

Richterich, P. (1989). Non-radioactive chemical sequencing of biotin labelled DNA. Nucleic Acids Research 17:21812186.

Ricterich, P. and Church, G.M. (1993). DNA sequencing with direct transfer electrophoresis and non-radioactive detection. Methods in Enzymology 218:187-222.

Rosenblum, B.B., Lee, L.G., Spurgeon, S.L., Khan, S.H., Menchen, S.M., Heiner, C.R. and Chen, S.M. (1997). New dye-labeled terminators for improved DNA sequencing patterns. Nucleic Acids Research 25:4500-4504.

Rosenthal, A., Sproat, B., Voss, H., Stegemann, J., Schwager, C., Erfle, H., Zimmermann, J., Coutelle, C. and Ansorge, W. (1990). Automated sequencing of fluorescently labelled DNA by chemical degradation. DNA Sequence 1: 63-71.

Sanger, F., Nicklen, S. and Coulson, A.R. (1977). DNA sequencing with chain terminator inhibitors. Proceedings of the National Academy of Sciences USA 74:5463-5467.

Schubert, F., Ahlert, K., Cech, D. and Rosenthal, A. (1990). One-step labelling of oligonucleotides with fluoresceine during automated synthesis. Nucleic Acids Research 18:3427.

Schubert, F., Cech, D., Reinhardt, R. and Wiesner, P. (1992). Fluorescent labelling of sequencing primers for automated oligonucleotide synthesis. DNA Sequence 2:273-279.

Smith, L.M., Fung, S., Hunkapillar, M.W., Hunkapillar, T.J. and Hood, L.E. (1985). The synthesis of oligonucleotides containing an aliphatic amino group at the 5' terminus: synthesis of fluorescent DNA primers for use in DNA sequence analysis. Nucleic Acids Research 13:2399-2412.

Smith, L.M., Sanders, J.Z., Kaiser, R.J., Hughes, P., Dodd, C.,Connell, C.R., Heiner, C., Kent, S.B.H. and Hood, L.E. (1986). Fluorescence detection in automated DNA sequence analysis. Nature 321:674-679.

Starke, H.R., Yan, J.Y., Zhang, J.Z., Mühlegger, K., Effgen, K. and Dovichi, N.J. (1994). Internal labeling with fluorescent deoxynucleotides in two-label peak-height encoded DNA sequencing by capillary electrophoresis. Nucleic Acids Research 22:3997-4001.

Steffens, D.L., Jang, G.Y., Sutter, S.L., Brumbaugh, J.A., Middendorf, L.R., Mühlegger, K., Mardis, E.R., Weinstock, L.A. and Wilson, R.K. (1995). An infrared fluorescent dATP for labeling DNA. Genome Research 5:393-399.

Tabor, S. and Richardson, C.C. (1995). A single residue in DNA polymerases of the Escherichia Coli DNA polymerase I family is critical for distinguishing between deoxy- and dideoxyribonucleotides. Proceedings of the National Academy of Sciences USA 92:6339-6343.

Theisen, P., McCollum, C. and Andrus, A. (1992). Fluorescent dye phosphoramidite labelling of oligonucleotides. Nucleic Acids Symposium Series 27:99100.

Voss, H., Nentwich, U., Duthie, S., Wiemann, S., Benes, V., Zimmermann, J. and Ansorge, W. (1997). Automated cycle sequencing with Taquenase: protocols for internal labeling, dye primer and "doublex" simultaneous sequencing. BioTechniques 23:312-318.

Voss, H., Schwager, C., Wirkner, U., Zimmermann, J., Erfle, H., Hewitt, N.A., Rupp, T., Stegemann, J. and Ansorge, W. (1991). A new procedure for automated DNA sequencing with multiple internal labelling by fluorescent dUTP. Methods in Molecular and Cellular Biology 3:30-34.

Wiemann, S., Rupp, T., Zimmermann, J., Voss, H., Schwager, C. and Ansorge, W. (1995). Primer design for automated DNA sequencing utilizing T7 DNA polymerase and internal labeling with fluorescein-15-dATP. BioTechniques 18:688-697.

Wiemann, S., Schilke, A., Rechmann, S., Zimmermann, J., Voss, H. and Ansorge, W. (1996). Reducing "double sequences" in automated DNA sequencing with T7 DNA polymerase and internal labeling. BioTechniques 20:791792.

Wiemann, S., Stegemann, J., Grothues, D., Bosch, A., Estvill, X., Schwager, C., Zimmermann, J., Voss, H. and Ansorge, W. (1995). Simultaneous on-line DNA sequencing of both strands with two fluorescent dyes. Analytical Biochemistry 224:117-121.

Wu, T., Ruan K. and Liu, W. (1996). A fluorescencelabeling method for sequencing small RNA on polyacrylamide gel. Nucleic Acids Research 24:3472-3473. 
A. Label on primer
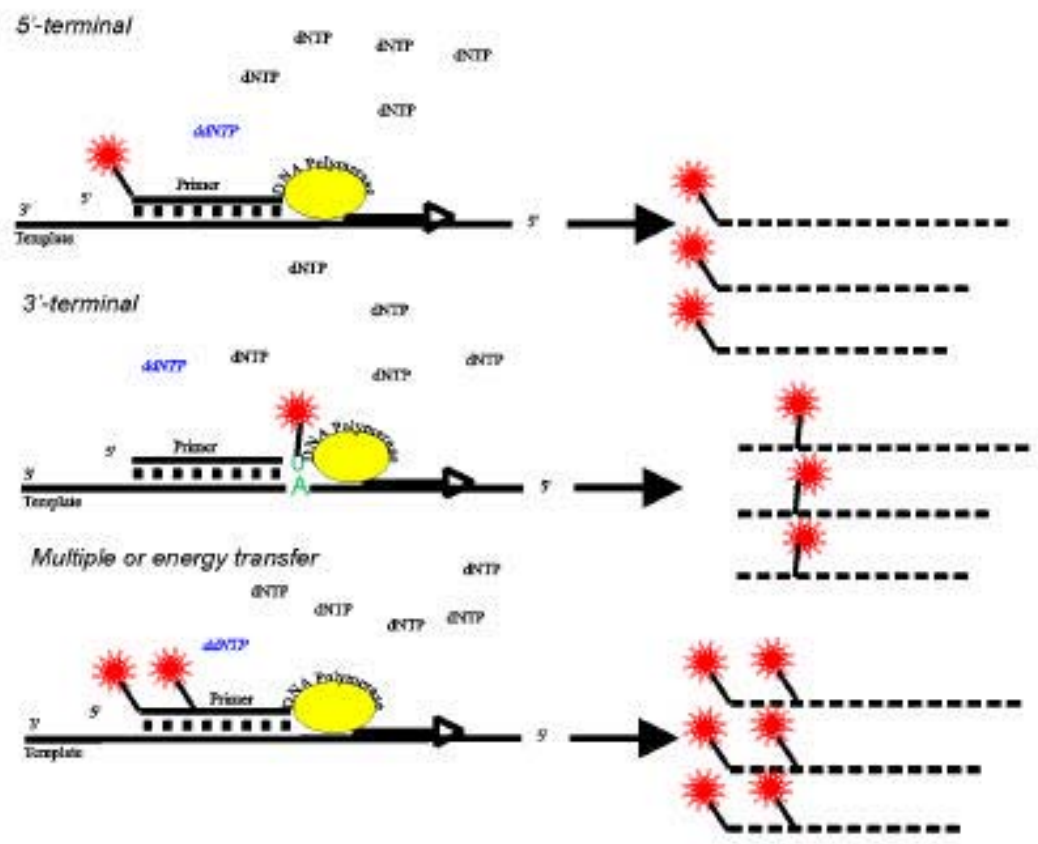

Enzyme-linked detection

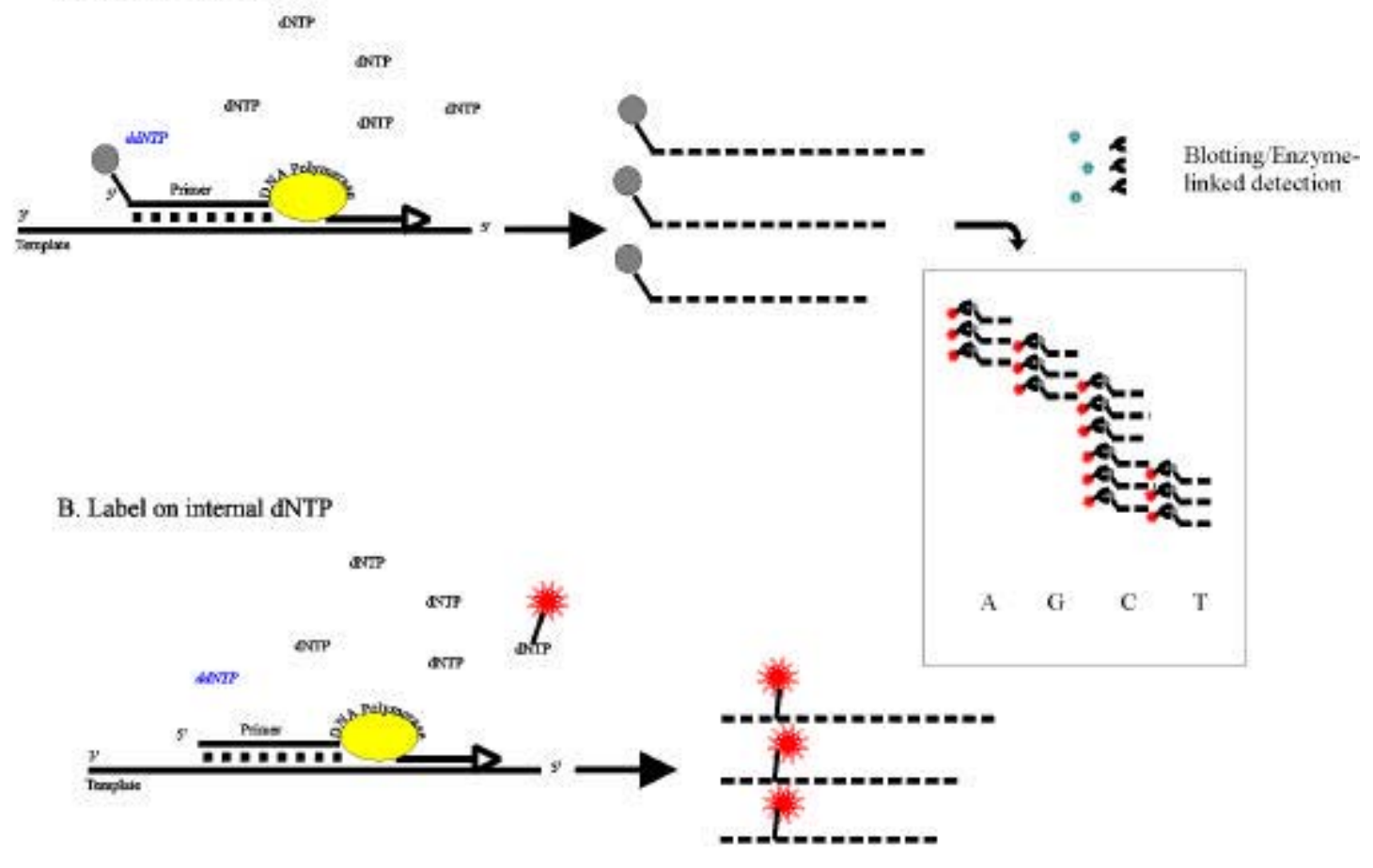

C. Label on $3^{4}$ dideoxy terminator

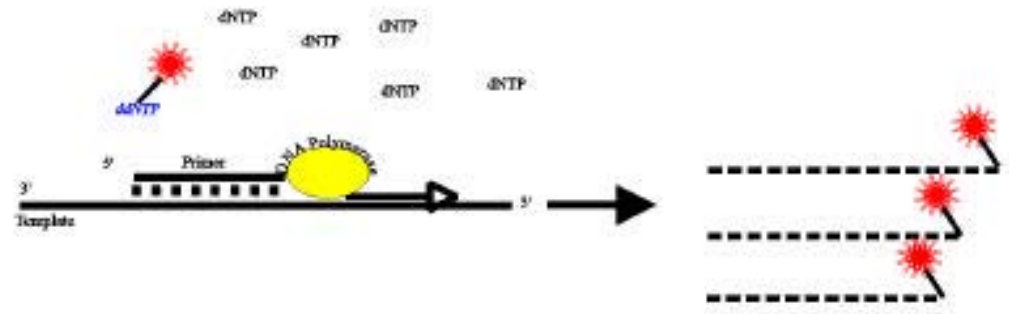

Fig. 1. Pathways for introducing a label during a DNA sequencing reaction.

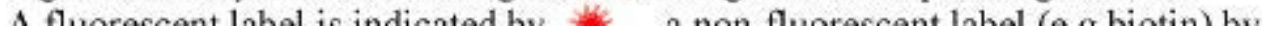

